\title{
A new Decision Based Median Filter using Cloud Model for the removal of high density Salt and Pepper noise in digital color images
}

\author{
K. Kannan, \\ Associate Professor, Department of Mechanical Engineering, \\ Kamaraj College of Engineering and Technology, Virudhunagar -626001, India. \\ E-mail: kannan_kcet@yahoo.co.in
}

\begin{abstract}
Removing the noise from digital color images plays a vital role in many of the image processing applications. Salt and Pepper noise is one type of the impulse noise which corrupts images during image capture or transmission or storage etc. This paper proposes and implements a new decision based median filter using cloud model to restore the highly corrupted digital color images. The proposed filter is tested on different images and shows better performance than standard median filter, adaptive median filter, decision based median filter and modified decision based median filter in terms of root mean square error, peak signal to noise ratio and image quality index.
\end{abstract}

Index Terms - Image Processing, Salt and Pepper noise, Cloud Model and Decision based Median Filter.

\section{INTRODUCTION}

Image Denoising is the process of removing the noise from the digital images using some prior knowledge about the noise while retaining as much as possible important image features. Basically, there are two approaches to image denoising based on the domain in which the denoising taken place. These approaches are named as spatial domain and transform domain filtering approaches. Spatial Filtering approaches remove the noise by manipulating the image in the spatial domain itself, whereas Transform Filtering approaches manipulate the image in transform domain.

Spatial filtering of images is an important aspect of image processing as it provides means for removing noise and sharpening blurred images. There are many types of spatial filters which can be classified into linear and non-linear filters. The simplest linear spatial filter is mean filter [1], which works by passing a mask over the image calculating the mean intensity and setting the central pixel to this value. They tend to remove the fine details in the image and fail to remove high level noise effectively. Among spatial filters, the famous non-linear filter is standard median filter (SMF) [2].

SMF is similar to mean filter but it replaces the centre pixel of the window by the median value of the window. The main drawback of SMF is that it exhibits blurring by removing the lines and corners in the image while suppressing the noise. To overcome this drawback, several variations of SMF have been proposed.

The weighted median filter (WMF) is one of the extensions to median filter which assigns more weights to some pixels in the window $[3,4]$. This WMF provides some degree of control to the smoothing behaviour through the weights assigned. These weights introduce additional complexity in the design and implementation of WMF. Another variation of SMF is the Centre Weighted Median Filter (CWMF), which gives more weights to the central value of the window only, thereby reduces the complexity in the design [5]. The CWMF filter performs well for low noise level and fails when the noise level is high. To overcome this, Adaptive Median Filters (AMF) with variable window size was introduced [6].

$\mathrm{AMF}$ is robust in removing the impulse noise while preserving the image details even though the probability of occurrence of impulse noise is high. The filters discussed above unconditionally replace each pixel with median value of the window without checking whether the pixel is "bad" or not. As a result, since the uncorrupted pixels are altered, they damage many image details in the high noise levels. To avoid the damage of uncorrupted pixels, the use of switching filters were introduced. These filters employ an impulse detector to determine the presence of pixels corrupted by impulses in the image. Only these noisy pixels will be filtered by these switching filters.

The Progressive Switching Median Filter (PSMF) is one of the switching filters in which both impulse detector and noise filter are applied progressively in iterative manner to obtain the best results [7]. These progressive iteration increases the time complexity of the filter. Again, in switching filters, the noisy pixels are replaced by median value without taking the local features into account. At higher noise densities, the median value of the window may also be a noisy pixel.

To overcome this problem, decision based median filter (DBMF) was proposed to replace the corrupted pixels by either the median pixel or left neighborhood pixel [8]. Although this filter shows promising results, smooth transition between the pixels is lost leading to degradation in the visual quality of the image. To provide smooth transition between the pixels with edge 
preservation and better visual quality, a modified decision based median filter (MDBMF) was proposed in which noisy pixel is replaced by the median pixel or the mean of the neighborhood processed pixels [9]. The noisy pixel replacement by the mean of the neighborhood processed pixels will lead to blurring of the edges and fine details in the image. To preserve the edges and fine details, this paper proposes and implements a new decision based median filter using cloud model to restore the highly corrupted digital color images. The proposed filter is tested on different images and shows better performance than standard median filter, adaptive median filter, decision based median filter and modified decision based median filter in terms of root mean square error, peak signal to noise ratio and image quality index.

The remaining portion of the paper is organized as follows. The second section of this paper discusses about cloud model and its parameters. The proposed new decision based median filter is described in third section. The evaluation criteria and the results of the proposed filter are presented in fourth and fifth section of this paper. The last section concludes the paper.

\section{CLOUD MODEL}

To remove the impulse noise in the digital images, it is necessary to grasp the impulse noise characteristics. Uncertainties are inherent features of impulse noise [10]. So, understanding the uncertainties and apply them in a better way can improve the performance of impulse noise removing filter. Uncertainties in impulse noise exist through the randomness and the fuzziness. The fact that the pixels are randomly corrupted and randomly set to the maximum or minimum extreme value shows the randomness whereas not all of the extreme value pixels are the noisy pixels shows the fuzziness associated with impulse noise. The relationship between the randomness and fuzziness was established by Cloud Model (CM) [11]. CM is a model of the uncertainty transformation between quantitative representation and qualitative concept based on normal distribution and bell shaped membership function. CM has been successfully applied to data mining [12, 14], image classification [13], image segmentation [15, 16] and optimization [17].

Let $U$ is a quantity domain expressed with accurate numbers and $\mathrm{C}$ is a quality concept in $\mathrm{U}$. If the quantity value, $x \in \mathrm{U}$ and $x$ is a random realization of the quality concept $\mathrm{C}$, then $\mu(x)$ is the membership degree of $x$ which lies between $[0,1]$. It is the random number which has the steady tendency,

$$
\mu: U \rightarrow[0,1], \forall x \in U, x \rightarrow \mu(x)
$$

The distribution of $x$ is called cloud and each $x$ is called a cloud drop. The cloud can be characterized by three parameters, i.e., the expected value $E_{x}$, entropy $E_{n}$, and hyperentropy $H_{e}[10-17] . \quad E_{x}$ is the expectation of the cloud drops' distribution. It points out which drops can best represent the concept and reflects the distinguished feature of the concept. $E_{n}$ is the uncertainty measurement of the qualitative concept, which is determined by both the randomness and the fuzziness of the concept. It represents the value region in which the drop is acceptable by the concept, while reflecting the correlation of the randomness and the fuzziness of the concept. $\mathrm{H}_{\mathrm{e}}$ is the uncertainty measurement of $\mathrm{E}_{\mathrm{n}}$. Given these three characteristics, a set of cloud drops can be generated with certainty degree by the normal cloud generator CG. Each pixel in the image is the cloud drop and composes the cloud. These cloud drops are given input to the backward cloud generator $\mathrm{CG}^{-1}$. The outputs of $\mathrm{CG}^{-1}$ are three parameters of cloud $\mathrm{E}_{\mathrm{x}}, \mathrm{E}_{\mathrm{n}}$ and $\mathrm{H}_{\mathrm{e}}$. This is shown in Figure 1.

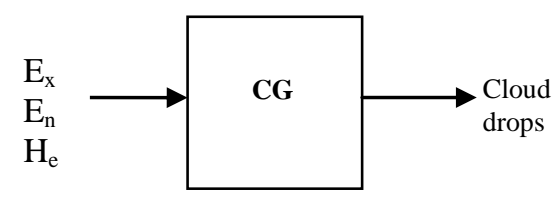

(a)

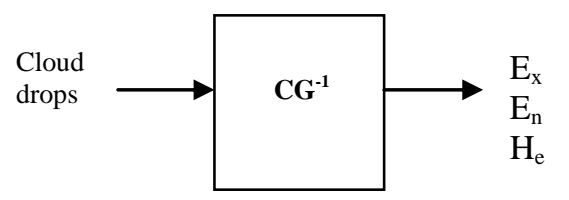

(b)

Figure 1. (a). Forward Cloud Generator (b). Backward Cloud Generator

When the drops are approaching the expected value $E_{x}$, the certainty degrees and the contribution degrees of the drops are increasing. Therefore, the drops in the cloud contribute to the concept with the different contribution degrees [20]. The drops located within $\left[\mathrm{E}_{\mathrm{x}}-\right.$ $3 \mathrm{E}_{\mathrm{n}}, \mathrm{E}_{\mathrm{x}}+3 \mathrm{E}_{\mathrm{n}}$ ] take up to $99.99 \%$ of the whole quantity and contribute $99.74 \%$ to the concept. Thus, the drops are located out of domain $\left[\mathrm{E}_{\mathrm{x}}-3 \mathrm{E}_{\mathrm{n}}, \mathrm{E}_{\mathrm{x}}+3 \mathrm{E}_{\mathrm{n}}\right]$, and their contributions to the concept can be neglected. This is "3En rule." According to the normal cloud generator (CG), the certainty degree of each drop is a probability distribution rather than a fixed value. It means that the certainty degree of each drop is a random value in a dynamic range. If $\mathrm{H}_{e}$ of the cloud is 0 , then the certainty degree of each drop will change to be a fixed value. The fixed value is the expectation value of the certainty degree. In fact, the value is also the unbiased estimation for the average value of the certainty degrees in the range. A curve called cloud expectation curve (CEC) can be constructed by plotting all the drops in $\mathrm{X}$-axis and their expectations of certainty degrees in $\mathrm{Y}$-axis. The CEC of cameraman image is shown in the Figure2. 


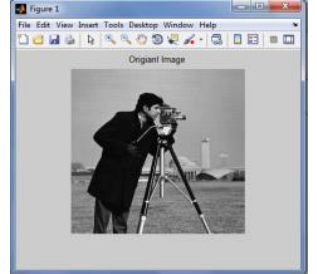

(a)

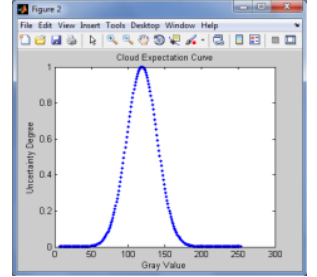

(b)
Figure 2. (a).Cameraman Image (b).CEC

\section{PROPOSED NEW DECISION BASED NEDIAN FILTER}

The proposed new decision based median filter (PNDBMF) is a two stage filter, where the first stage is the noisy pixel detector and the second stage is the noisy pixels replacement filter. In the first stage, each pixel is tested to find whether the pixel is noisy or noise-free. When a pixel is classified as noise-free, it will be retained and the filtering action is avoided without altering any fine details and textures that are contained in the original image. When a noisy pixel is detected in the first stage, then the median value of the window is also tested. If the median value of the window is not noisy, then the noisy pixel is replaced by median value of the window. Otherwise, the noisy pixel is replaced by the value ' 0 ' and passed to the next stage for filtering.

\section{A. Noisy Pixel Detector}

Similar to other impulse detection algorithm, this Noisy Pixel Detector (NPD) uses prior information about the impulsive noise with the following assumptions.

- Only the proportions of image pixels are corrupted while other pixels are noise free.

- Noisy pixels take a very large value as positive impulse or a very small value as negative impulse.

Normally, the impulse noise is modeled as salt and pepper noise. The salt noise takes the pixel value of 255 and pepper noise takes the pixel value of 0 . These two pixel values are used to identify the noisy pixels in the image. The NPD checks the value of every pixel in the image. If the pixel value is ' 0 ' or ' 255 ', the pixel is classified as noisy pixel. Otherwise the pixel is classified as noise free pixel. When a pixel is classified as noise free, it will be retained without altering any fine details and textures in the original image. When a noisy pixel is detected in the first stage, then the median value of the window is also tested. If the median value of the window is not noisy, then the noisy pixel is replaced by median value of the window. Otherwise, the noisy pixel is replaced by the value ' 0 ' and passed to the next stage for filtering.

\section{B. Noisy Pixel Replacement Filter}

The Noisy Pixel Replacement Filter (NPRF) replaces the noise pixel with the value ' 0 ' by the weighted fuzzy mean value of the remaining pixels in the square filtering window $W_{i, j}^{2 N+1}$ of size $2 N+1$. $\mathrm{W}_{\mathrm{i}, \mathrm{j}}^{2 \mathrm{~N}+1}=\left\{\mathrm{x}_{\mathrm{i}+\mathrm{s}, \mathrm{j}+\mathrm{t}}\right\}$, where $\mathrm{s}, \mathrm{t} \in(-\mathrm{N}, \ldots 0, \ldots \mathrm{N})$.

Step 1: Set the window size by initializing $\mathrm{N}=1$. Then, $\mathrm{E}_{\mathrm{x}}$ of each uncorrupted pixels in $W_{i, j}^{2 N+1}$ is calculated using the formulae,

$$
E_{X}=\frac{1}{n} \sum_{x_{i+s, j}+t \in W_{i, j}^{2 N+1}} x_{i+s, j+t}
$$

Step 2: Calculate $\mathrm{E}_{\mathrm{n}}$ using the following formulae,

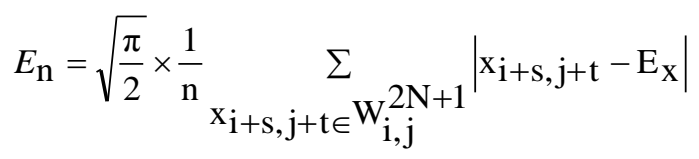

Step 3: Calculate weights for $\mathrm{x}_{\mathrm{i}+\mathrm{s}, \mathrm{j}+\mathrm{t}}$

$$
\mathrm{w}_{\mathrm{i}+\mathrm{s}, \mathrm{j}+\mathrm{t}}=\exp \left(-\left(\mathrm{x}_{\mathrm{i}+\mathrm{s}, \mathrm{j}+\mathrm{t}}-\mathrm{E}_{\mathrm{X}}\right)^{2} / 2 \mathrm{E}_{\mathrm{n}}^{2}\right)
$$

Step 4: Calculate the weighted mean

$$
Y_{i, j}=\sum_{x_{i+s, j+t \in W_{i, j}^{2 N+1}}} w_{i+s, j+t} x_{i+s, j+t} / w_{i+s, j+t}
$$

Step 5: Replace the noisy pixel value by weighted mean $Y_{i, j}$.

\section{EVALUATION CRITERIA}

The following evaluation measures are used in this paper. The Root Mean Square Error (RMSE) between the reference image $R$ and fused image $F$ is given by,

$R M S E=\sqrt{\frac{\sum_{i=1}^{N} \sum_{j=1}^{N}[R(i, j)-F(i, j)]^{2}}{N^{2}}}$

The Peak Signal to Noise Ratio (PSNR) between the reference image $\mathrm{R}$ and fused image $\mathrm{F}$ is given by,

$\mathrm{PSNR}=10 \log _{10}(255)^{2} /(\mathrm{RMSE})^{2}(\mathrm{db})$

Quality index of the reference image $(\mathrm{R})$ and fused image $(\mathrm{F})$ is given by [18],

$\mathrm{QI}=\frac{4 \sigma_{a b} \mathrm{ab}}{\left(\mathrm{a}^{2}+\mathrm{b}^{2}\right)\left(\sigma_{\mathrm{a}}^{2}+\sigma_{b^{2}}\right)}$ 
The maximum value $\mathrm{Q}=1$ is achieved when two images are identical, where $\mathrm{a} \& \mathrm{~b}$ are mean of images, $\sigma_{a b}$ be covariance of $\mathrm{R} \& \mathrm{~F}, \sigma_{a}{ }^{2}, \sigma_{b}{ }^{2}$ be the variance of image R,F.

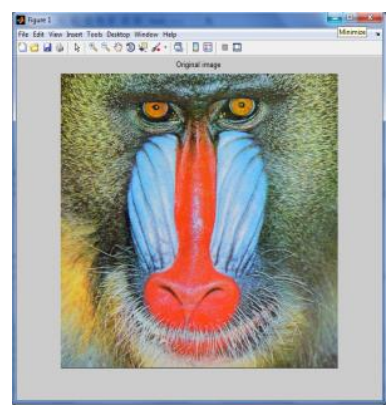

a. Mandrill

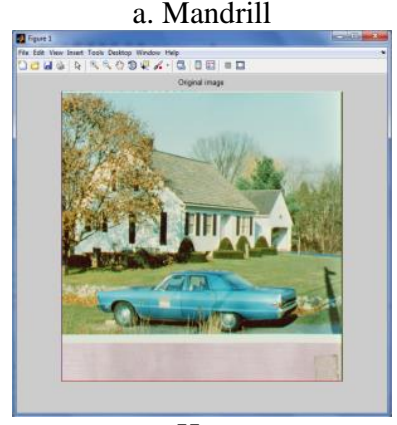

c. House

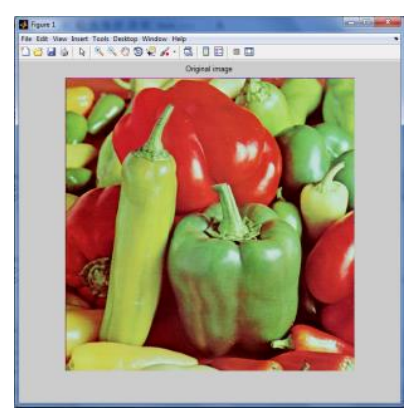

b. Pepper

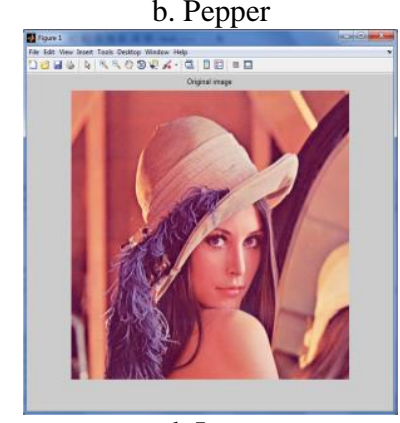

d. Lena
Figure 3. Original Images

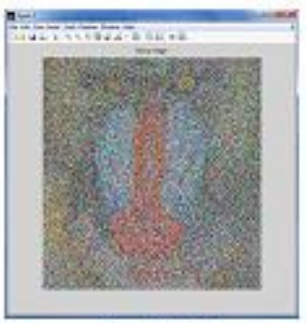

a. Noiny Imsso

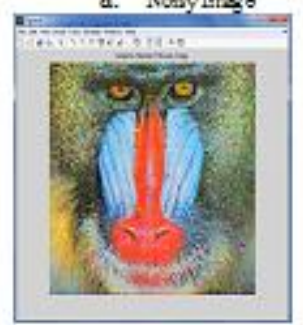

C. ANG Inago

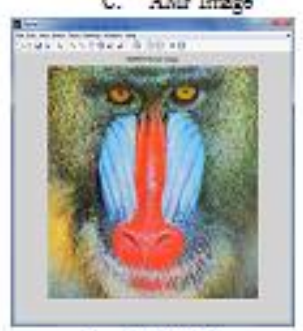

e. MDENG hago
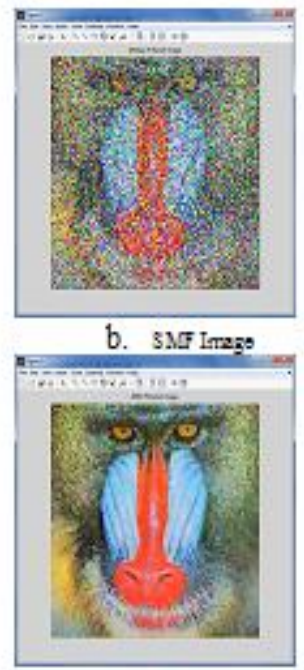

d. DBMT Insgo

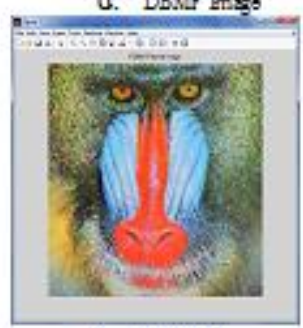

f. RNDMT Insgo
Figure 4. Results of various filtered Images for mandrill image of noise density $75 \%$
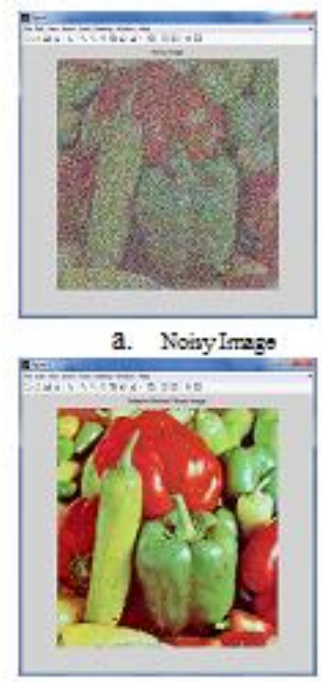

C. ANG Image

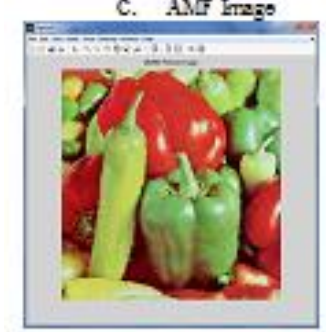

e. MDEMI Imago

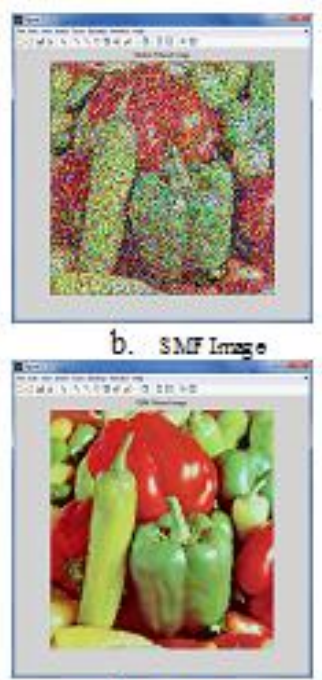

d. DEMT Imago

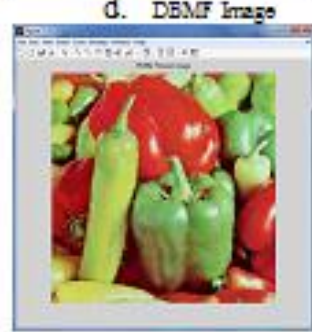

f. PNDBNG Imago

Figure 5. Results of various filtered Images for pepper image of noise density $75 \%$
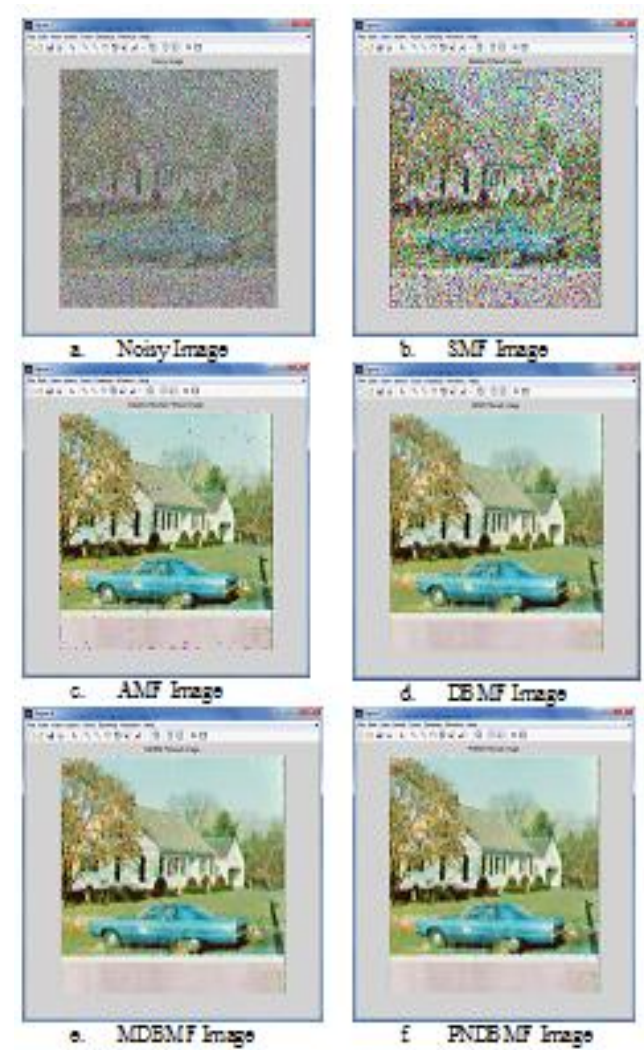

Figure 6. Results of various filtered Images for house image of noise density $75 \%$ 
50 A new Decision Based Median Filter using Cloud Model for the removal of high density Salt and Pepper noise in digital color images

TABLE I. PERFORMANCE COMPARISON TABLE FOR MANDRILL IMAGE AT DIFFERENT NOISE DENSITIES

\begin{tabular}{|l|c|c|c|c|c|}
\hline \multicolumn{7}{|c|}{ Noise } & 0.75 & 0.8 & 0.85 & 0.9 & 0.95 \\
\hline Noisy & 120.72 & 124.651 & 128.502 & 132.129 & 135.699 \\
\hline SMF & 94.1488 & 103.638 & 112.849 & 121.871 & 130.55 \\
\hline AMF & 31.5887 & 38.2727 & 49.7289 & 68.0547 & 95.6429 \\
\hline DBMF & 29.1042 & 31.405 & 34.3988 & 38.3776 & 44.8895 \\
\hline MDBMF & 28.803 & 30.9906 & 33.843 & 37.4185 & 43.4878 \\
\hline PNDBMF & 28.7143 & 30.7673 & 33.3327 & 36.1653 & 40.1523 \\
\hline \multicolumn{7}{|c|}{ PSNR } \\
\hline Noisy & 6.4968 & 6.2186 & 5.954 & 5.7123 & 5.4807 \\
\hline SMF & 8.6557 & 7.8224 & 7.0818 & 6.4141 & 5.8165 \\
\hline AMF & 18.1559 & 16.4803 & 14.1999 & 11.4772 & 8.5193 \\
\hline DBMF & 18.8755 & 18.214 & 17.4231 & 16.4754 & 15.1166 \\
\hline MDBMF & 18.9655 & 18.3288 & 17.5666 & 16.6941 & 15.3894 \\
\hline PNDBMF & 18.9926 & 18.3907 & 17.6978 & 16.9899 & 16.0863 \\
\hline \multicolumn{7}{|c|}{0.89} \\
\hline Noisy & 0.0941 & 0.0721 & 0.0512 & 0.0341 & 0.0172 \\
\hline SMF & 0.2526 & 0.1866 & 0.1293 & 0.0818 & 0.041 \\
\hline AMF & 0.8313 & 0.7658 & 0.6428 & 0.4572 & 0.2287 \\
\hline DBMF & 0.8511 & 0.8267 & 0.7928 & 0.7453 & 0.6538 \\
\hline MDBMF & 0.8536 & 0.8304 & 0.7979 & 0.7545 & 0.6692 \\
\hline PNDBMF & 0.8549 & 0.8333 & 0.8046 & 0.771 & 0.7169 \\
\hline
\end{tabular}

TABLE II. PERFORMANCE COMPARISON TABLE FOR PEPPER IMAGE AT DIFFERENT NOISE DENSITIES

\begin{tabular}{|c|c|c|c|c|c|}
\hline Noise & 0.75 & 0.8 & 0.85 & 0.9 & 0.95 \\
\hline \multicolumn{6}{|c|}{ RMSE } \\
\hline Noisy & 122.586 & 129.247 & 133.346 & 137.073 & 140.795 \\
\hline SMF & 94.0307 & 105.908 & 116.306 & 125.656 & 135.137 \\
\hline AMF & 23.9472 & 29.2673 & 44.6285 & 66.0374 & 97.3284 \\
\hline DBMF & 22.2268 & 20.4416 & 24.274 & 30.3079 & 40.3 \\
\hline MDBMF & 22.0389 & 20.0305 & 23.7984 & 29.4482 & 39.0779 \\
\hline PNDBMF & 21.76 & 19.4516 & 22.2675 & 26.7154 & 33.6652 \\
\hline \multicolumn{6}{|c|}{ PSNR } \\
\hline Noisy & 6.3622 & 5.9081 & 5.637 & 5.3978 & 5.1648 \\
\hline SMF & 8.6655 & 7.637 & 6.8241 & 6.1529 & 5.5205 \\
\hline AMF & 20.5486 & 18.825 & 15.1546 & 11.7506 & 8.3746 \\
\hline DBMF & 21.2015 & 22.0745 & 20.593 & 18.7214 & 16.2463 \\
\hline MDBMF & 21.2755 & 22.2516 & 20.7576 & 18.9477 & 16.4749 \\
\hline PNDBMF & 21.388 & 22.5026 & 21.3278 & 19.7599 & 17.7644 \\
\hline \multicolumn{6}{|c|}{ QI } \\
\hline Noisy & 0.089 & 0.0702 & 0.0491 & 0.0322 & 0.017 \\
\hline SMF & 0.2642 & 0.1978 & 0.1329 & 0.0856 & 0.0436 \\
\hline AMF & 0.8979 & 0.8536 & 0.7027 & 0.4933 & 0.2464 \\
\hline DBMF & 0.909 & 0.9266 & 0.8966 & 0.8417 & 0.7177 \\
\hline MDBMF & 0.9104 & 0.9295 & 0.8999 & 0.8484 & 0.7279 \\
\hline PNDBMF & 0.9129 & 0.9334 & 0.9123 & 0.874 & 0.7988 \\
\hline
\end{tabular}


TABLE III. PERFORMANCE COMPARISON TABLE FOR HOUSE IMAGE AT DIFFERENT NOISE DENSITIES

\begin{tabular}{|c|c|c|c|c|c|}
\hline Noise & 0.75 & 0.8 & 0.85 & 0.9 & 0.95 \\
\hline \multicolumn{6}{|c|}{ RMSE } \\
\hline Noisy & 122.599 & 126.645 & 130.468 & 134.295 & 137.961 \\
\hline SMF & 94.0083 & 104.105 & 113.763 & 123.371 & 132.602 \\
\hline $\mathrm{AMF}$ & 24.189 & 31.6178 & 45.1788 & 65.492 & 95.1604 \\
\hline DBMF & 22.2148 & 25.0528 & 28.5946 & 34.2598 & 43.7103 \\
\hline MDBMF & 22.0201 & 24.7395 & 28.2373 & 34.0619 & 43.281 \\
\hline PNDBMF & 21.7035 & 24.1939 & 26.9062 & 31.5053 & 37.8527 \\
\hline \multicolumn{6}{|c|}{ PSNR } \\
\hline Noisy & 6.3614 & 6.0793 & 5.8211 & 5.5699 & 5.336 \\
\hline SMF & 8.6676 & 7.7816 & 7.0111 & 6.3068 & 5.6802 \\
\hline $\mathrm{AMF}$ & 20.4606 & 18.1338 & 15.0343 & 11.8073 & 8.5629 \\
\hline DBMF & 21.2054 & 20.1557 & 19.0128 & 17.4385 & 15.3236 \\
\hline MDBMF & 21.2821 & 20.2657 & 19.1228 & 17.4891 & 15.4117 \\
\hline PNDBMF & 21.4085 & 20.4604 & 19.5399 & 18.1676 & 16.5722 \\
\hline \multicolumn{6}{|c|}{ QI } \\
\hline Noisy & 0.0891 & 0.0685 & 0.0501 & 0.0319 & 0.0158 \\
\hline SMF & 0.2646 & 0.1967 & 0.1395 & 0.0861 & 0.0431 \\
\hline AMF & 0.8957 & 0.8308 & 0.6953 & 0.4885 & 0.2449 \\
\hline DBMF & 0.9089 & 0.8848 & 0.8506 & 0.7854 & 0.656 \\
\hline MDBMF & 0.9103 & 0.8873 & 0.8534 & 0.7861 & 0.6557 \\
\hline PNDBMF & 0.913 & 0.8928 & 0.8676 & 0.8185 & 0.7438 \\
\hline
\end{tabular}

TABLE IV. PERFORMANCE COMPARISON TABLE FOR HOUSE IMAGE AT DIFFERENT NOISE DENSITIES

\begin{tabular}{|c|c|c|c|c|c|}
\hline Noise & 0.75 & 0.8 & 0.85 & 0.9 & 0.95 \\
\hline \multicolumn{6}{|c|}{ RMSE } \\
\hline Noisy & 121.47 & 125.599 & 129.46 & 133.198 & 136.924 \\
\hline SMF & 92.7514 & 103.163 & 112.9 & 122.374 & 131.823 \\
\hline $\mathrm{AMF}$ & 17.7353 & 26.9811 & 41.8173 & 63.2114 & 94.4781 \\
\hline DBMF & 14.0953 & 16.7304 & 19.5351 & 24.0599 & 32.7897 \\
\hline MDBMF & 13.8682 & 16.3605 & 19.1632 & 23.4925 & 32.6373 \\
\hline PNDBMF & 13.5161 & 15.864 & 18.0859 & 21.1386 & 26.3262 \\
\hline \multicolumn{6}{|c|}{ PSNR } \\
\hline Noisy & 6.4472 & 6.1569 & 5.8937 & 5.6465 & 5.4075 \\
\hline SMF & 8.7911 & 7.8668 & 7.0822 & 6.3826 & 5.7382 \\
\hline AMF & 23.1732 & 19.5159 & 15.7075 & 12.1209 & 8.6329 \\
\hline DBMF & 25.1803 & 23.7122 & 22.3739 & 20.5713 & 17.89 \\
\hline MDBMF & 25.3207 & 23.9051 & 22.5437 & 20.7648 & 17.929 \\
\hline PNDBMF & 25.5403 & 24.1699 & 23.0471 & 21.6808 & 19.8115 \\
\hline \multicolumn{6}{|c|}{ QI } \\
\hline Noisy & 0.0672 & 0.0514 & 0.0364 & 0.0234 & 0.0114 \\
\hline SMF & 0.2101 & 0.1521 & 0.1035 & 0.0632 & 0.0288 \\
\hline $\mathrm{AMF}$ & 0.921 & 0.8297 & 0.6582 & 0.4286 & 0.198 \\
\hline DBMF & 0.9483 & 0.9285 & 0.9025 & 0.8534 & 0.7302 \\
\hline MDBMF & 0.9499 & 0.9314 & 0.9062 & 0.8583 & 0.7322 \\
\hline PNDBMF & 0.9523 & 0.9352 & 0.9164 & 0.8847 & 0.8227 \\
\hline
\end{tabular}


52 A new Decision Based Median Filter using Cloud Model for the removal of high density Salt and Pepper noise in digital color images

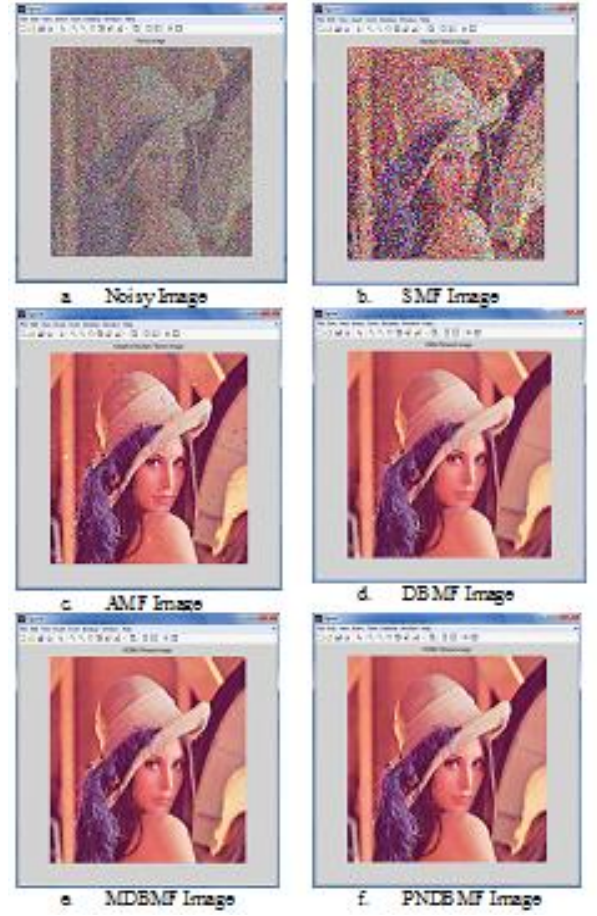

Figure 7. Results of various filtered Images for lena image of noise density $75 \%$
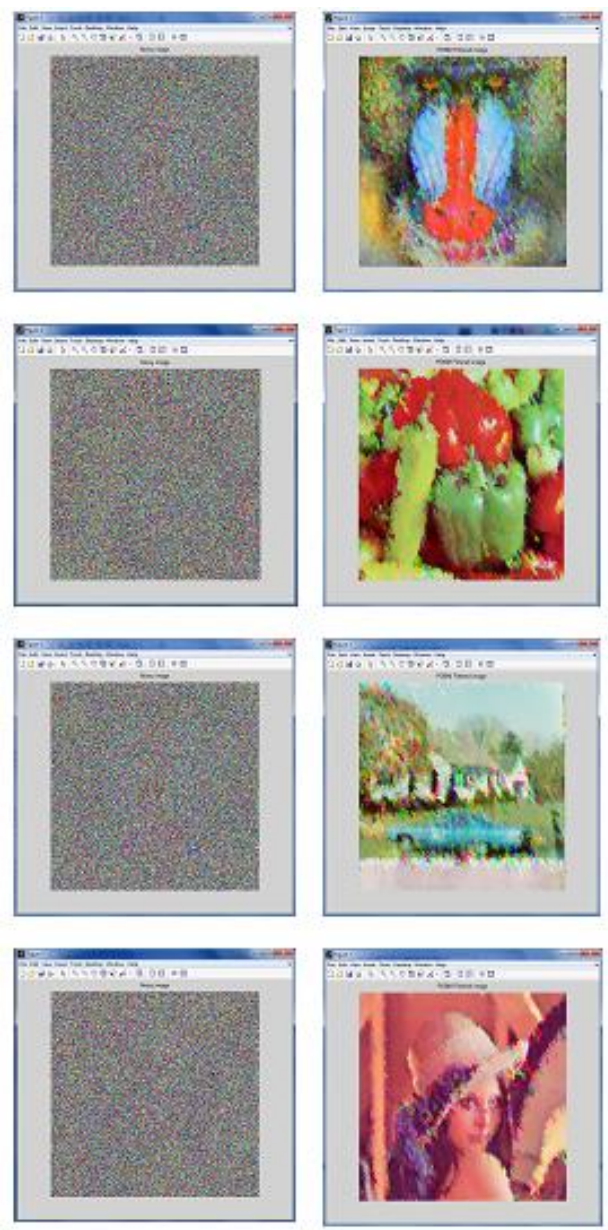

Figure 8. Results of PNDBMF Images at $95 \%$ of Noise density Column I - Noisy Images \& Column II - PNDBMF Images

\section{RESULTS}

For simulation, four color test images of size $512 \mathrm{X}$ 512 namely mandrill, pepper, house (in tiff format) and lena (in jpeg format) are taken and shown in Figure 3. To this image, salt \& pepper noise is added with noise level varying from $75 \%$ to $95 \%$ with increments of $5 \%$. In this work, it is assumed that the images are corrupted by $\mathrm{P} \%$ salt \& pepper noise in which the salt is made up of $0.5 \mathrm{P} \%$ and pepper is made up of $0.5 \mathrm{P} \%$. The restoration results of SMF, AMF, DBMF, MDBMF and PNDBMF for the noise level of 0.75 for various images are shown in Fig. 4 - 7. Table 1- 4 shows the performance of above filters in terms of RMSE, PSNR \& QI for the above test images. From the results, it is inferred that only FMF and WFMF are able to produce reconstructed images with good image detail preservation. However, the proposed WFMF has a better noise suppression ability in terms of RMSE, PSNR and QI.

\section{CONCLUSIONS}

In this paper, a new decision based median filter for removal of high density salt and pepper has been proposed and implemented. This filter represents the uncertainties of the salt and pepper noise perfectly by using cloud model, which is essential in removing the noise. In addition, the above filter identifies the noise pixel directly, without any need to sort the pixel gray values, which immensely increases the computational efficiency in noise detection. Even if the noise density is close to 0.95 , the texture, details and edges of the images are preserved with good visual effect as shown in figure 7. In total, the proposed filter is a moderately simple denoising filter with good detail preservation.

\section{ACKNOWLEDGMENT}

The author thanks anonymous referees for their comments and suggestions.

\section{REFERENCES}

[1] Kundu, S. K. Mitra, and P. P. Vaidyanathan. Application of two dimensional generalized mean filtering for removal of impulse noises from images. IEEE Trans, 1984, ASSP-32(3): 600-609.

[2] Thomas S. Huang, George J. Yang and Gregory Y. Tang. A Fast Two-Dimensional Median Filtering Algorithm. IEEE Trans, 1979, ASSP-27(1):13-18.

[3] L. Yin, R. Yang, M. Gabbouj, and Y. Neuvo. Weighted median filters: A tutorial. IEEE Trans, 1996, Circuits and Systems II, Analog and Digital Signal Processing, 43(3): 157-192.

[4] D. Brownrigg. The weighted median filter. Commun. Assoc. Comput., 1984, 27(8): 807-818.

[5] S. J. Ko and S. J. Lee. Center weighted median filters and their applications to image enhancement. 
IEEE Trans, 1991, Circuits and Systems, 38(9): 984 $-993$.

[6] H. Hwang and R. A. Haddad. Adaptive median filters: New algorithms and results. IEEE Trans, 1995, Image Processing, 4(4): 499-502.

[7] Z. Wang and D. Zhang. Progressive switching median filter for the removal of impulse noise from highly corrupted images. IEEE Trans, 1999, Circuits and Systems II, Analog Digital Signal Processing, 46(1): 78-80.

[8] K. S. Srinivasan and D. Ebenezer. A new fast and efficient decision based algorithm for removal of high-density impulse noises. IEEE Signal Processing Letter, 2007, 14(3): 189-192.

[9] Madhu S. Nair, K. Revathy and Rao Tatavarti. Removal of Salt-and Pepper Noise in Images: A New Decision-Based Algorithm. Proc. of International Multi Conference of Engineers and Computer Scientists, IMECS 2008: 19-21.

[10] Zhe Zhou. Cognition and Removal of Impulse Noise with Uncertainty. IEEE Trans, 2012, Image Processing, 21(7): 3157-3167.

[11] Deyi Li, Changyu Liu and Wenyan Gan. A New Cognitive Model: Cloud Model. International Journal of Intelligent Systems, 2009, 24: 357-375.

[12] H. J. Wang and Y. Deng. Spatial clustering method based on cloud model. Proc. of IEEE International Conference on Fuzzy Systems and Knowledge Discovery, 2007, 2: 272-276.

[13] Y.L. Qi. Classification for trademark image based on normal cloud model. Proc. of IEEE International Conference on Information Management, Innovation Management and Industrial Engineering, 2009, 3: 74-77.

[14] H. Chen and B. Li. Qualitative rules mining and reasoning based on cloud model. Proc. of IEEE International Conference on Software Engineering and Data Mining, 2010, 523-526.

[15] K. Qin, K. Xu, Y. Du, and D. Y. Li. An image segmentation approach based on histogram analysis utilizing cloud model. Proc. of IEEE International Conference on Fuzzy Systems and Knowledge Discovery, 2010, 2: 524-528.

[16] Y. Q. Shi and X. C. Yu. Image segmentation algorithm based on cloud model the application of fMRI. Proc. of IEEE International Conference on Intelligent Computation Technology and Automation, 2008, 2: 136-140.

[17] Y. Gao. An optimization algorithm based on cloud model. Proc. of IEEE International Conference on Computational Intelligence and Security, 2009, 2: 84-87.

[18] Zhou Wang and Alan C. Bovik. A Universal Image Quality Index. IEEE Signal Processing Letters, 2002, 9(3): 81-84.

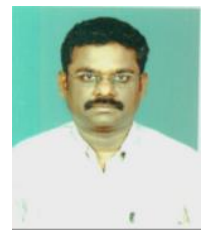

K. Kannan received the Bachelor of Engineering Degree in Electronics and Communication Engineering from Madurai Kamaraj University in the year 1992. Also, he received Master of Engineering Degree in Industrial Engineering Degree from Anna University in the year 2004. Currently he is doing his $\mathrm{Ph} . \mathrm{D}$ in the area of Digital Image Processing. He has more than fourteen years of teaching experience and currently working as Associate Professor in the department of Mechanical Engineering of Kamaraj College of Engineering and Technology, Virudhunagar. His area of interest includes Signal Processing, Image Processing, Embedded System and RTOS. So far he published more than fifty technical papers in national and international journals and conferences. He is a life member of ISTE, IETE, BES and IACSIT.

How to cite this paper: K. Kannan,"A new Decision Based Median Filter using Cloud Model for the removal of high density Salt and Pepper noise in digital color images", IJIGSP, vol.6, no.4, pp.46-53, 2014.DOI: 10.5815/ijigsp.2014.04.06 